\title{
Application of Locality Preserving Projections in Face Recognition
}

\author{
Shermina.J $J^{\#,}$ \\ \# Department of Computing, Muscat College, University of Stirling, UK, \\ Shermina@muscatcollege.edu.om
}

\begin{abstract}
Face recognition technology has evolved as an enchanting solution to address the contemporary needs in order to perform identification and verification of identity claims. By advancing the feature extraction methods and dimensionality reduction techniques in the application of pattern recognition, a number of face recognition systems has been developed with distinct degrees of success. Locality preserving projection (LPP) is a recently proposed method for unsupervised linear dimensionality reduction. LPP preserve the local structure of face image space which is usually more significant than the global structure preserved by principal component analysis (PCA) and linear discriminant analysis (LDA). This paper focuses on a systematic analysis of locality-preserving projections and the application of LPP in combination with an existing technique This combined approach of LPP through MPCA can preserve the global and the local structure of the face image which is proved very effective. Proposed approach is tested using the AT \& T face database. Experimental results show the significant improvements in the face recognition performance in comparison with some previous methods.
\end{abstract}

Keywords- Image Processing; Face Recognition; Image Compression; Multilinear Systems; Locality preserving projection

\section{INTRODUCTION}

Biometric technologies have been evolved as an enchanting solution to perform secure identification and personal verification. The need for highly secure identification and personal verification technologies is becoming apparent as the level of security breaches and transaction fraud increases. The increasing use of biometric technologies in high security applications and beyond has created the requirement for highly dependable face recognition systems. Face recognition system is used to verify an identity of a person by matching a given face against a database of known faces. It has become an alternative to the traditional identification and authentication methods such as the use of keys, ID cards and passwords.

Face recognition involves computer recognition of personal identity based on geometric or statistical features that are derived from the face images [2],[7],[8]. Even though human can detect and identify faces in a scene easily, building an automated system is challenging. Face recognition technology can be applied to a wide variety of application areas including access control for PCs, airport surveillance, private surveillance, criminal identification and for security in
ATM transaction. In addition, face recognition system is moving towards the next-generation smart environment where computers are designed to interact more like humans [12].

In recent years, considerable progress has been made in the area of face recognition with the development of many techniques. In the recent past, face recognition research has witnessed a growing interest in subspace analysis techniques [2],[7]. Two of the classical algorithms, principal component analysis (PCA) and linear discriminant analysis (LDA)[2], which are well-known for feature extraction and dimension reduction, has been widely used in face recognition. However, both PCA and LDA effectively see only the linear manifold that based on the Euclidean structure. They fail to capture the underlying structure which lies on a nonlinear submanifold hidden in the image space. Recently, some nonlinear methods have been developed to discover the nonlinear structure of the manifold, algorithms e.g. Isomap, locally linear embedding (LLE), and Locality Preserving Projections (LPP)[3],[7] in which the first two algorithms are nonlinear but the LPP algorithm is a linear dimensionality reduction algorithm.

PCA aims to preserve the global structure of the face image space and the LDA method aims to preserve the discriminating information, but the goal of LPP method is to preserve the local structure of image samples. Locality Preserving Projections describe face images by mapping the face data onto a low-dimensional face feature subspace called "Laplacianfaces". The advantages of LPP algorithm are that LPP is a linear method and it preserve the local information of the face image space. The limitation of LPP is that it represents an image by a vector in high-dimensional space which is often confronted with the difficulty that sometimes the image matrix is singular. It is unsupervised and hence some of the important information with the classification are neglected. To overcome the complication of the singular problem, Laplacianface method projects the image set onto a PCA subspace to confirm to be non-singular.

In this paper, we are projecting the face data onto a Multilinear Principal Component Analysis (MPCA) subspace, and LPP algorithm is further used to preserve the local structure information. This combined approach considering the global and local structure of the face image space can obtain a more effective optimal subspace for face representation and recognition. First, it compresses and preserves the principal information in a matrix form, so it removes more inherent redundancy, and a much lower 
dimensional face representation is acquired by which the recognition speed is enhanced greatly. Second, the low dimensional represent exempts the consequent LPP step from the singularity problem and it also achieves a more competitive accurate recognition rate than the Laplacianface. The organization of the paper is as follows: Literature review is presented in Section 2. Description about the face recognition techniques used in our research is presented in section 3. Methodology of the approach is presented in Section 4. Experimental results and the comparative analysis is given in section 5 and finally the conclusions are summed up in Section 6.

\section{LITERATURE REVIEW}

Many face recognition methods have been developed in the past few decades. Most common feature extraction methods are principal component analysis (PCA)[1] and linear discriminant analysis (LDA) [2]. Another linear technique which is used for face recognition is Locality Preserving Projections (LPP) [3],[4], which finds an embedding that preserves local information, and gains a face subspace that best detects the essential face manifold structure[9],[11]. Xiaofei He introduced the Locality Preserving Projections (LPP) as when the high dimensional data lies on a low dimensional manifold embedded in the ambient space, the Locality Preserving Projections are obtained by finding the optimal linear approximations to the eigen functions of the Laplace Beltrami operator on the manifold. Because of this, LPP, being linear, shares many of the data representation properties of nonlinear techniques such as Laplacian Eigenmaps or Locally Linear Embedding.

Lin Kezheng et al [13] proposed the Enhanced Locality Preserving Projections, to identify the underlying manifold structure of a data set. ELPP considers both the between-class scatter and the within-class scatter in the processing of manifold learning. Equivalently, the goal of ELPP is to preserve the within-class geometric structure, while maximizing the between-class distance. Zhonglong Zheng et al.[18] proposed supervised locality preserving projection (SLPP), using class labels of data points to enhance its discriminant power in their mapping into a low dimensional space. The GSLPP method, which is robust to variations of illumination and facial expression, applies the SLPP to an augmented Gabor feature is vector derived from the Gabor wavelet representation of face images.

$\mathrm{Yu}$ Weiwei et al. [17] proposed two-dimensional discriminate locality preserving projections (2D-DLPP), which benefits from three techniques, i.e., locality preserving projections (LPP), image based projection and discriminant analysis. Deng Cai et al. [14] have proposed an appearance based face recognition method, called orthogonal Laplacian face in which face data may be generated by sampling a probability distribution that has support on or near a submanifold of ambient space. Earlier works based on PCA or LDA [2] suffer from not preserving the local manifold of the face structure whereas the research works on LPP lacks to preserve global features of face images[3]. Some papers [3], [7] uses the combination of both PCA and LPP, captures only the most expressive features.

Yi Jin et al. [5] presented a new manifold learning algorithm in which a bilateral-projection-based 2DPCA (B2DPCA) for image matrix compression is performed before supervised locality preserving projections. The bilateralprojection-based DPCA algorithm is used to obtain the meaningful low dimensional structure of the data space. Also those works that uses PCA captures the variation in the samples without considering the variance among the subjects. The combination of global feature extraction technique LDA and local feature extraction technique LPP to achieve a high quality feature set called Combined Global and Local Preserving Features (CGLPF) that captures the discriminate features among the samples considering the different classes in the subjects which produces the considerable improved results in facial image representation and recognition[12].

Motivated by this research, our approach combines the global feature preservation technique MPCA and the local feature preservation technique LPP to form the high quality feature set. The concept is to project the face data to an MPCA space for preserving the global information and then projecting to Locality Preserving Projection (LPP) space by using the distance preserving spectral methods, to add the local neighbourhood manifold information. Analysing these techniques, a unique feature extraction technique is not felicitous when the dimensionality of face images attempts to reach its peek and combination of two feature extraction methods on distinctly separate subspaces seems to be more effective in the performance of face recognition.

\section{METHODOLOGY \\ A. Locality Preserving Projections}

Locality Preserving Projections (LPP) are linear projective maps that arise by solving a variational problem that optimally preserves the neighbourhood structure of the data set. LPP represents a linear approximation of the nonlinear Laplacian eigenmaps introduced in [3]. When high-dimensional data lies on a low dimension manifold embedded in the data space, then LPP approximate the eigenfunctions of the LaplaceBeltrami operator of the manifold. LPP aims at preserving the local structure of the data. This is unlike PCA and LDA, which aims at preserving the global structure of the data. LPP is unsupervised and performs a linear transformation. It models the manifold structure by constructing an adjacency graph, which is a graph expressing local nearness of the data. This is highly desirable for face recognition compared to nonlinear local structure preserving, since it is significantly less computationally expensive and more importantly it is defined in all points and not just in the training points as Isomaps and Laplacian Eigenmaps. Let $x i, i=1,2, \cdots, n$, denote the training patterns of $m$ classes. We use $X=[x 1, x 2, \cdots, x n]$ to denote the data matrix and use $l(x i)$ to denote the label of $x i$, say, $l(x i)=k$ implies that $x i$ belongs to class $k$. LPP aims at preserving the intrinsic geometry of the data by forcing 
neighboring points in the original data space to be mapped into closely projected data. The algorithm starts by defining a similarity matrix $\mathbf{W}$, based on a (weighted) $k$ nearest neighbors graph, whose entry $\mathbf{W} i j$ represents the edge between training images (graph nodes) $\mathbf{x} i$ and $\mathbf{x} j$. Gaussiantype weights of the form $W_{i j}=e^{-\frac{\left\|x_{i}-x_{j}\right\|^{2}}{t}}$ have been proposed in [23], although other choices (e.g., cosine type) are also possible. Based on matrix $\mathbf{W}$, a special objective function is constructed, enforcing the locality of the projected data points by penalizing those points that are mapped far apart. Basically, the approach reduces to finding a minimum eigenvalue solution to the generalized eigenvalue problem.

\section{B. Algorithm}

Locality Preserving Projection (LPP) is one of the linear approximation obtained from the nonlinear Laplacian Eigenmap [3]. The algorithmic procedure of LPP is stated below:

1) Construction of adjacency graph: Let $G$ denote a graph with $m$ nodes and an edge between nodes $i$ and $j$, if $x_{i}$ and $x_{j}$ are close. There are two variations:

(a) $\varepsilon$-neighbourhoods: Nodes $i$ and $j$ are connected by an edge if $\left\|x_{i}-x_{j}\right\|^{2}<\varepsilon$, where the norm is the usual Euclidean norm in $R^{n}$.

(b) $k$ nearest neighbors: Nodes $i$ and $j$ are connected by an edge if $i$ is among $k$ nearest neighbors of $j$ or $j$ is among $k$ nearest neighbors of $i$.

2) Choosing the weights: We have two variations for weighting the edges. $W$ is a sparse symmetric $m \times m$ matrix with $W_{i j}$ having the weight of the edge joining vertices $i$ and $j$, and 0 if there is no such edge.

(a) Heat kernel: If nodes $i$ and $j$ are connected, $W_{i j}=e^{-\frac{\left\|x_{i}-x_{j}\right\|^{2}}{t}}$

(b) Simple-minded: $W_{i j}=1$, if and only if vertices $i$ and $j$ are connected by an edge.

3) Eigenmaps: Compute the eigenvectors and eigenvalues for the generalized eigenvector problem:

$$
X L X^{T} a=\lambda X D X^{T} a
$$

Where $D$ is a diagonal matrix whose entries are column (or row, since $W$ is symmetric) sums of $W$,
$D_{i i}=\sum_{j} W_{j i} \cdot L=D-W$ is the Laplacian matrix. The $i^{\text {th }}$ column of matrix $X$ is $x_{i}$.

Let the column vectors $a_{0}, \cdots, a_{l-1}$ be the solutions of equation (1), ordered according to their eigenvalues, $\lambda_{0}<\cdots<\lambda_{l-1}$. Thus, the embedding is as follows:

$$
x_{i} \rightarrow y_{i}=A^{T} x_{i}, A=\left(a_{0}, a_{1}, \cdots, a_{l-1}\right)
$$

Where $y_{i}$ is a $l$-dimensional vector and $A$ is a $n \times l$ matrix.

\section{Implementation}

Initially, image processing techniques such as normalization and resizing of the face images are employed in preprocessing in order to improve the face image since variations in lighting conditions dramatically decrease recognition performance. When processing a face, the features like variations in light, image quality, persons' pose, facial expressions are taken into account. Normalization is used to outweigh any illumination variations or relative sizes between two sets of faces. The face images in the database are usually of different dimensions. Therefore the input samples necessitate to be resized to standard dimensions. Hence, image pre-processing and normalization is the important part of face recognition systems. Next, the feature extraction is achieved by merging the MPCA along with LPP to calculate the feature projection matrices. MPCA receives the set of face image samples of the same dimensions as input for feature extraction. The resultant output of the MPCA is the dimensionally reduced feature projection matrix of face images. The dimension reduced feature projection matrices of face image samples obtained using MPCA is then fed as an input to the LPP algorithm. Locality Preserving Projection (LPP) is one of the linear approximation obtained from the nonlinear Laplacian Eigenmap [9]. The dimensional reduced feature matrices of the training sample images obtained using the MPCA and LPP techniques are stored in a database. While we are testing the face images, the aforesaid techniques are applied to generate the feature matrix and thereby a similarity measure is carried out on the sample face images. The face recognition can be done by comparing the test feature matrix with the enrolled face features in the database using L2 distance that measures the query images with the nearest database images. The similarity distance measure for a pair of face images is computed in which a threshold determines whether the pair of face is identified as similar or not.

\section{COMPARATIVE ANALYSIS}

Existing experiments on AT\&T facial images formerly called as ORL database were analyzed and the performance is observed as per the procedures applied to AT\&T facial images [15]. Accuracy in face recognition is to computed with the 
false acceptance rate (FAR) which is percentage of incorrect acceptances and false rejection rate (FRR) which is the percentage of incorrect rejections. The genuine acceptance rate (GAR) is computed using these factors and the overall accuracy measurement of the proposed approach is calculated using the formula $100-(F A R / F R R) / 2$ which is shown in Fig. 1. The comparative results of existing appearance based methods in face recognition were analyzed and found that the performance of LPP supersedes the PCA and LDA approaches. Moreover, the latest experimental results show that the combination of LPP with MPCA improves the accurate recognition rate in face recognition. The table clearly shows the efficiency of LPP in comparable with the other approaches. The comparative recognition rate of various approaches is shown in the following table1.

TABLE I

Comparison of recognition rates on AT\&T databases

\begin{tabular}{|c|c|c|}
\hline \multirow{2}{*}{$\begin{array}{l}\text { Data } \\
\text { base }\end{array}$} & \multicolumn{2}{|c|}{ Performance Comparisons } \\
\hline & Method & $\begin{array}{l}\text { Accurate } \\
\text { Recognition Rate }\end{array}$ \\
\hline \multirow{4}{*}{$\begin{array}{l}\stackrel{5}{2} \\
\stackrel{4}{4}\end{array}$} & PCA(Eigenface) & $88.50 \%$ \\
\hline & LDA(Fisherface) & $91.00 \%$ \\
\hline & LPP(Laplacianface) & $94.00 \%$ \\
\hline & MPCA + LPP & $96.5 \%$ \\
\hline
\end{tabular}

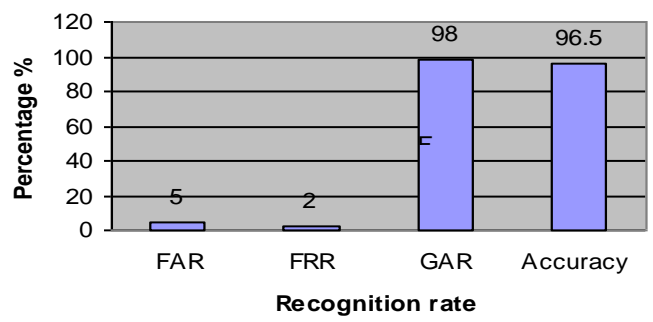

Face Recognition is the active area of research over the past two decades which have resulted in successful techniques for recognizing the facial images. In this article, the importance of Locality Preserving Projection is studied and the comparison of existing face recognition technique with the impact of LPP is also analysed. The combined appearance based technique such as LPP with MPCA outperforms the previously developed locality preserving projection approach to yield a good recognition rate. With these results, we conclude that the combination of LPP with any other approaches could improve the recognition accuracy. Therefore, in future work, the combination of LPP with other appearance based approaches could be experimented for accurate recognition rate in order to
[10] Haiping Lu, K.N. Plataniotis and A.N. Venetsanopoulos, "MPCA Multilinear Principal Component Analysis of Tensor Objects", IEEE Transactions on Neural Networks, Vol. 19, No. 1, January 2008.

[11] Zhao, W. Chellappa, R., Phillips, P. J. and Rosenfeld, A., "Face Recognition: A Literature Survey", ACM Computing Survey, December, pp: 399-458, 2003.

[12] Ruba.S.Kathavarayan and Murugesan Karuppasamy, "Preserving Global and Local Features for Robust Face Recognition under Various Noisy Environments", International Journal of Image Processing, Volume(3), Issue(6)

[13] Lin Kezheng, Lin Sheng and Chen Dongmei: "Enhanced Locality

Preserving Projections". CSSE (1) 2008: 985-988
Deng Cai, Xiaofei He, Jiawei Han and Hong-Jiang Zhang,

\section{ACKNOWLEDGMENT}

I would like to thank Dr.V.Vasudevan for providing the guidance in the preliminary discussions in order to proceed further in my area of research.

\section{REFERENCES} P.N. Belhumeur, J.P. Hepanha, and D.J. Kriegman, "Eigen faces vs.fisherfaces: recognition using class specific linear projection" no. 7, pp. 711-720, 1997 . MA: MIT Press, 2003. - 2376, 2007 M. Turk and Pentland, "Face Recognition Using Eigenfaces", in Proc IEEE International Conference on Computer Vision and Pattern Recognition, Maui, Hawaii, 1991. Transactions on Pattern Analysis and Machine Intelligence, vol. 7., No. 3, March 2005 Information Processing Systems 14, Vancouver, British Columbia, Canada, 2002. "Orthogonal Laplacianfaces for Face Recognition", IEEE Transactions On Image Processing, 2006

"AT\&T database of faces" \{Online Available:http://www.cl.cam.ac.uk/ research/dtg/attarchive/ facedatabase.html

[16] Zhonglong Zheng, Fan Yang, Wenan Tan, Jiong Jia and Jie Yang, "Gabor feature-based face recognition using supervised locality preserving projection”, Signal Processing Volume 87, Issue 10, October 2007, Pages 2473-2483

[17] $\mathrm{Yu}$ Weiwe "Two-dimensional discriminant locality preserving projections for face recognition" Pattern Recognition Letter Volume 30, Issue 15, 1 November 2009, Pages 1378-1383 determine the most efficient approach in appearance based

(International Symposium on 